\title{
Influence of Fines on the Surface Energy Heterogeneity of Lactose for Pulmonary Drug Delivery
}

\author{
Raimundo Ho, ${ }^{a}$ Adrian S. Muresan, ${ }^{b}$ Gerald A. Hebbink, ${ }^{c}$ and Jerry Y. Y. Heng ${ }^{\mathrm{a},}{ }^{*}$ \\ ${ }^{a}$ Surfaces and Particle Engineering Laboratory, Department of Chemical Engineering, Imperial \\ College London, South Kensington Campus, London SW7 2AZ, United Kingdom \\ ${ }^{\mathrm{b}}$ FrieslandCampina Research, Harderwijkerstraat 41006, 7418 BA Deventer, The Netherlands \\ ${ }^{\mathrm{c}}$ FrieslandCampina DOMO, Hanzeplein 25, 8017 JD, Zwolle, The Netherlands \\ * Corresponding author: Tel: +44 (0)20 7594 0784; Fax: +44 (0)20 75945638. \\ E-mail address: jerry.heng@imperial.ac.uk (Jerry Y.Y. Heng)
}




\begin{abstract}
The effects of the blending of lactose fines to the overall adhesion property of coarse $\alpha$-lactose monohydrate carrier particles were investigated. Five samples, three of them commercial samples from DOMO (Lactohale ${ }^{\circledR}$ LH100, LH210, and LH250) whilst the other two are blends of LH210 and LH250, were studied. Characterisation included particle sizing, SEM, PXRD and IGC. Dispersive surface energy $\gamma_{S V}^{d}$ was determined using a finite concentration IGC method to obtain a distribution profile. The $\gamma_{S V}^{d}$ distribution of lactose crystals were found to vary from 40$48 \mathrm{~mJ} / \mathrm{m} 2$. The unmilled coarse crystalline lactose sample (LH100) $\gamma_{S V}^{d}$ was lowest and showed less heterogeneity than the milled sample (LH250). Fines (LH210) were found to have the highest $\gamma_{S V}^{d}$ value. The samples with loaded LH210 were found to have a higher energy than LH100. The amount of LH210 in Blend 1 was not able to decrease surface energy heterogeneity, whereas sample Blend 2 showed adequate loading of fines to obtain a relatively homogeneous surface. Addition of fines resulted in an increase in $\gamma_{S V}^{d}$, suggesting that coarse lactose surfaces were replaced by surfaces of the fines. Increasing the loading of fines may result in a more homogeneous surface energy of lactose particles.
\end{abstract}

Keywords: Carrier Particle; Lactose; Pulmonary Drug Delivery; Inverse Gas Chromatography; Surface Energy. 


\section{Introduction}

Pulmonary drug delivery is an attractive way of introducing medication for lung diseases like asthma and COPD. Besides, pulmonary delivery is increasingly used for delivery of drugs in medications that cannot be swallowed like proteins and peptides (Del Valle et al., 2009). The lungs are very attractive for the uptake of medications due to their large surface area ( $70-100$ $\mathrm{m}^{2}$ for an adult) and their easily permeable membrane. In order to reach the active area of the lungs, drug particles need to be in the aerodynamic particle size range of $1-5 \mu \mathrm{m}$. These small particles tend to form strong agglomerates caused by cohesive forces and these forces need to be overcome to produce an aerosol with particles that are able to reach the lungs. In dry powder inhalers, a coarse carrier, most commonly lactose monohydrate, is blended with the micronised drug particles. Current literature reports that upon inhalation, an aerosol is produced where the fine drug particles are detached from the coarse carrier particles.

The performance of carrier-based dry powder inhalation (DPI) formulation is determined, to a significant degree, by the preparation of the formulation, as well as the design of aerosolisation device, the inhalation mode and the respiratory-tract anatomy and physiology of the patient (Timsina et al., 1994). One of the methods, that have been extensively researched, to optimise drug delivery of the active ingredient is the use of a ternary formulation where a small amount of fine excipient particles or fines is added to the coarse carrier and the drug blend (Podczeck, 1998; Louey and Stewart, 2002; Shur et al., 2008). Despite the evidence that the use of such methods show an improved fine particle dose (FPD) or fine particle fraction (FPF) of the active drug, the mechanism by which the fine particles alters the performance of the formulation has remained elusive (Jones and Price, 2006). 
One such hypothetical mechanism that has been proposed is the passivation of strong binding sites or high energy sites by fine excipient particles (Jones and Price, 2006). The fines are thought to preferentially adhere to the surface regions of the coarse carrier with the highest surface energy, therefore forcing drug particles to bind to surfaces with lower surface energy. When the formulation is kinetically activated through the respiratory-tract, the drug particles are more easily separated from the surface of the carrier, thus increasing the inhaled dose in the lower airways (Fig. 1). Zeng et al. (1999) observed an increase of $\sim 120 \%$ in FPF of the drug when fine lactose particles were blended to the coarse lactose carrier before the addition of drug to the formulation, thus providing the fines with the first opportunity to adhere to the high energy sites. Other blending order was found to yield a smaller increase in FPF compared to the control, i.e. the binary mixture of coarse carrier and drug. However, it was also noted that the blending order had no significant impact on the formulation performance when the blending time was increased, speculatively due to the redistribution of particles between binding sites for prolonged blending time (Zeng et al., 2000).

It is clear that with the current state of knowledge, the influence of fine particles to improve DPI formulation will require a more fundamental understanding of the interparticulate interactions of the components in the mixture, in order to clarify the mechanism by which the fines work (Jones and Price, 2006). In an attempt to understand the change in the overall adhesion property when lactose fines are added to the coarse carrier, this study seeks to determine the surface energetic distribution of different processed coarse lactose and the subsequent changes to the surface energy distributions by the blending of fine lactose particles. Such a study will confirm (or otherwise) the commonly and widely accepted view that addition 
of fines leads to a reduction in surface energy. This was conducted via a recently developed methodology using inverse gas chromatography (IGC) at finite concentrations.

\subsection{Inverse gas chromatograhy (IGC)}

The inverse gas chromatography technique has been widely used in the characterisation of solids, fibres, films including pharmaceutical solids (Heng et al., 2006). By measuring the time a probe molecule takes to elute through a packed column of sample materials, the thermodynamic properties of the solid phase can be determined. The net retention volume, $V_{N}$ which is a fundamental surface thermodynamic property of the solid-vapour interaction process, is given by:

$$
V_{N}=\frac{j}{m} \cdot F \cdot\left(t_{R}-t_{0}\right) \cdot \frac{T}{273.15}
$$

where $T$ is the column temperature in Kelvin $(\mathrm{K}), F$ is the carrier gas exit flow rate at 1 atm and 273.15 K, $t_{R}$ is the retention time for adsorbing probe and $t_{0}$ is the mobile phase hold-up time (dead-time), $m$ is the sample mass in the packed column and $j$ is the James-Martin correction, which corrects the retention time for the pressure drop along the column bed. $V_{N}$ can be related to the free energy of adsorption, $\Delta G^{0}$, and the work of adhesion of vapour adsorbates to the surface, $W_{A}$, by:

$$
-\Delta G^{0}=R T \ln V_{N}+K=N_{A} \cdot a_{m} \cdot W_{A}
$$

where $R$ is the universal gas constant, $K$ is a constant, $N_{A}$ is the Avogadro's number, $a_{m}$ is the cross sectional area of the adsorbate.

According to Owens and Wendt (1969), the work of adhesion between a solid and liquid is the summation of geometric mean terms of dispersive and polar surface energy components as 
shown in Eq. 3:

$$
W_{A}=2 \sqrt{\gamma_{L V}^{d} \cdot \gamma_{S V}^{d}}+2 \sqrt{\gamma_{L V}^{p} \cdot \gamma_{S V}^{p}}
$$

Hence, the injection of a series of infinite concentration of non-polar $\left(\gamma_{L V}^{p}=0\right)$ probes into the sample column will result in a linear regression on a plot of $R T \ln V_{N}$ versus $a_{m}\left(\gamma_{L V}^{d}\right)^{1 / 2}$, and $\gamma_{S V}^{d}$ can be calculated from the slope (Schultz et al., 1987) (Fig. 2). The polar component of surface energy can also be determined by applying a polar adsorbate $\left(\gamma_{L V}^{p} \neq 0\right)$ and as such, the $V_{N}$ measured is summation of a dispersive and polar component. Knowing $\gamma_{L V}^{d}$ of the polar probe allows the polar (also cited in literature as acid-base) energy of adsorption, $\Delta G_{A B}^{0}$, to be obtained.

IGC has been used to access the performance of dry powder inhalation formulations (Cline and Dalby, 2002; Tong et al., 2006). Tong et al. (2006) investigated the relative influence of drug-drug cohesion and drug-carrier adhesion on the in vitro performance of salmeterol xinafoate (drug) with lactose (carrier) by measuring surface energies and solubility parameters of the components by IGC at infinite dilution (zero surface coverage). The inhaler performance of salmeterol xinafoate was found to improve significantly if the drug-carrier adhesion was stronger than drug-drug cohesion. Cline and Dalby (2002) similarly observed that increasing surface energy interaction between drug and carrier resulted in an improved FPF of the drug.

\subsection{Surface free energy distribution}

A recently developed method (Thielmann et al., 2007) using inverse gas chromatography at finite concentration has been shown to be able to distinguish surfaces energetics of pharmaceutical solids exhibiting markedly different surface properties, i.e. heterogeneity and 
homogeneity (Ho et al., 2009a), as well as small variation in surface energy distributions resulting from small changes in crystal habits (Ho et al., 2009b). In this study, the methodology was utilised to characterise surface energetic profiles of different inhalation grade lactose, and the effects to the surface energetics when they are mixed in a two component blend were also investigated.

The measurement of dispersive and polar surface energy distribution relies on a series of finite concentration IGC experiments using a series of n-alkanes and at least one polar solvent. In the current study, the determination of $\gamma_{S V}^{d}$ and $\Delta G_{A B}^{0}$ distribution profiles consists of three main steps which are displayed in Fig. 3. First, the adsorption isotherms for four n-alkane probes (C6C10) and ethanol are measured using the IGC. Employing the peak maximum methodology (Thielmann et al., 2007), the retention volume and equilibrium partial pressure, $P$, are calculated for each single injection of increasing solute concentration via Eq. 1 and 4:

$$
P=\frac{h}{F \cdot A} \cdot V_{\text {Loop }} \cdot \frac{273.15}{T_{\text {Loop }}} \cdot \frac{P}{P_{0}}
$$

where $h$ is the chromatogram peak height, $A$ is the chromatogram peak area, $V_{\text {Loop }}$ is the injection loop volume, $T_{\text {Loop }}$ is the injection loop temperature and $P_{0}$ is the saturation pressure. The adsorbed amount, $n$, and therefore the adsorption isotherm for each probe vapour can then be obtained by integration of $V_{N}$ versus $P$. If the Brunauer-Emmett-Teller (BET) specific surface area, $S S A_{B E T}$, of the sample is known, the monolayer capacity, $n_{m}$, for each probe vapour can be calculated from:

$$
S S A_{B E T}=a_{m} \cdot N_{A} \cdot n_{m}
$$

From the monolayer capacity, the corresponding surface coverage, $n / n_{m}$ or $\theta$, at each injection concentration can then be measured from the amount adsorbed $n$. Since the retention volumes of 
each probe are now expressed as a function of sample surface coverage, both $\gamma_{S V}^{d}$ and $\Delta G_{A B}^{0}$ can now be calculated at a particular surface coverage in the same way as described previously in Fig. 2 using the Schultz approach (1987). The calculations of $\gamma_{S V}^{d}$ and $\Delta G_{A B}^{0}$ across a range of surface coverages, therefore, result in a distribution of dispersive and polar surface energies. Further details of the methodology can be found elsewhere (Thielmann et al., 2007; YlaMaihaniemi et al., 2008). 


\section{Materials and methods}

\subsection{Materials}

Details of the five lactose samples are tabulated in Table 1. Three of them are commercial samples from DOMO (Lactohale ${ }^{\circledR}$ LH100, LH210, and LH250), the other two are blends of LH210 and LH250. LH100 are coarse lactose crystals that were sieved through a $310 \mu \mathrm{m}$ sieve, LH210 and LH250 are milled and classified grades of lactoses, with LH210 being a fine fraction and LH250 being a coarse fraction.

Blends 1 and 2 were prepared by weighing the appropriate amounts of LH210 and LH250 in a bottle and turning and rotating the closed bottles for $>1$ minute. Blending ratios are for Blend 1: $82 \mathrm{~g}$ of LH250 and $18 \mathrm{~g}$ of LH210, and for Blend 2: $70 \mathrm{~g}$ of LH250 and $30 \mathrm{~g}$ of LH210.

\subsection{Particle size measurement}

Particle size distributions were measured with a Sympatec HELOS laser diffractometer

(Sympatec GmbH, Clausthal-Zellerfeld, Germany). All powders were dispersed in air at a pressure of 1.5 bar through the detection zone of the diffractometer. The diffracted laser light was focussed with an R5 lens with a measuring range of $0.5 / 4.5-875 \mu \mathrm{m}$.

\subsection{Finite concentration inverse gas chromatography}

Lactose powder (2.5-2.8 g) samples were packed by gentle vibration into separate presilanised glass columns $(300 \mathrm{~mm} \times 4 \mathrm{~mm}$ ID) with silanised glass wool packing at each end to prevent powder movement. The experiments were conducted using an SMS-iGC 2000 (Surface Measurement Systems, London, UK) system. Prior to measurements samples were pre-treated at 
$303 \mathrm{~K}$ and $0 \% \mathrm{RH}$ for 2 hours under flowing helium to remove any residual moisture or solvent adsorbed on the powder surface. Following pre-treatment, pulse injections using a $0.25 \mathrm{ml}$ gas loop at $303 \mathrm{~K}$ were performed. Purely dispersive n-alkane vapour probes (decane, nonane, octane and heptane) and polar probe (ethanol) were injected at $0.03,0.05,0.10,0.20,0.35,0.55,0.70$, $0.80,0.95 P / P_{0}$ and $V_{N}$ was determined using a peak maximum analysis. Methane gas was used as a non-interacting probe giving $t_{0}$ for the column, with concentrations of $0.10 P / P_{0}$. Helium was used as the carrier gas at a flow rate of 10.0 standard cubic centimetres per minute for all injections. The $\gamma_{S V}^{d}$ and $\Delta G_{A B}^{0}$ were calculated according to the Schultz method (1987).

\subsection{Specific surface area}

The BET specific surface area for all five lactose samples was obtained based on $\mathrm{N}_{2}$ adsorption isotherm measured using a gas adsorption analyzer (Tristar 3000, Micromeritics, Norcross, GA). Approximately $4 \mathrm{~g}$ of each sample was degassed with helium flow at $50^{\circ} \mathrm{C}$ for at least 5 hours before BET measurement. Surface area was determined using the BET model in the $P / P_{0}$ range from 0.05 to 0.30 . Each sample was measured in duplicate.

\subsection{SEM images}

SEM images were acquired with a tabletop microscope system TM-1000 (Hitachi, Tokyo, Japan) in the charge-up reduction mode. 


\subsection{Polymorph identification}

X-ray powder diffraction spectra were obtained for the lactose samples using a X'Pert Pro diffractometer (PANalytical B.V., Almelo, The Netherlands) over the range of $7-40^{\circ} 2 \theta$ with a $\mathrm{CuK} \alpha \mathrm{X}$-ray source at $40 \mathrm{kV}$ and $40 \mathrm{~mA}$. 


\section{Results and discussion}

Particle size and BET specific surface area of LH100, LH210, LH250, Blend 1 and Blend 2 are displayed in Table 2, whereas the SEM images are shown in Fig. 4. From Table 2, the fine lactose (LH210) has the smallest median-averaged equivalent spherical diameter $(18 \mu \mathrm{m})$ amongst the three unblended lactose samples. The unmilled sample LH100 has the largest median-averaged diameter, whereas the gently milled and classified LH250 possesses a smaller median-averaged diameter than LH100 due to the size reduction procedure. The particle sizes of Blend 1 and 2 are both between the median-averaged diameter of LH250 and LH210, and are also consistent with the blending ratio of LH250 to LH210. As can be seen from Fig. 4, LH100 exhibits the normal tomahawk crystal shape expected for $\alpha$-lactose monohydrate crystals (Raghavan et al., 2001). On the other hand, the crystal shape of the gently milled LH250, was found to be more irregular and it seems that the difference in crystal shapes (habit) between LH100 and LH250 could be due to the cleavage of weakly attached facets upon milling. The particle size of Blend 1, which was blended with 18\% fine lactose, is approximately $20 \%$ bigger than Blend 2 which contains the same batch of LH250 blended with $30 \%$ fines. Post-blending operations, the fines can be seen to adhere onto particular surface regions of both Blend 1 and Blend 2. However, some portions of fine particles also seem to form into clusters or aggregates which are adhered to the coarse crystal surfaces of Blend 1 and Blend 2. The amount of fines, which is defined here as the fraction of particles below $15 \mu \mathrm{m}$, follow the same trend as the median particle size: the smaller the median size, the larger the percentage of fine particles (Table 2). The coarse LH100 and LH250 only have about 3\% of fine particles, whereas the fine LH 210 has over $40 \%$ of fines. The two blends can also be seen to have quantities of fines that are 
there in between LH210 and LH250. The trend in BET specific surface areas, which are also displayed in Table II, is in good agreement with the median-averaged particle size of the samples. Comparisons of $\gamma_{S V}^{d}$ and $\Delta G_{A B}^{0}$ distributions for the five lactose samples are shown in Fig. 5 and Fig. 6 respectively. All unblended lactose, LH210, LH250 and LH100 show a small degree of dispersive surface energy heterogeneity within the range of surface coverage, $\theta$, examined (Fig. 5), although very different $\gamma_{S V}^{d}$ values. As the fines content of the unblended samples increases, the range of $\gamma_{S V}^{d}$ shifts to higher values. This means that the finer lactose crystals, in particular the LH210 fines, possess a greater degree of long-ranged van der Waals surface forces compared to the coarse lactose LH100 and LH250.

$\beta$-lactose can be present in milled materials, attributed to the recrystallisation of amorphous lactose during storage (Haque and Roos, 2005). In order to understand the variation in $\gamma_{S V}^{d}$ for these three lactose samples, polymorphic identification was conducted by PXRD. The PXRD patterns of both coarse lactose (LH100 and LH210), the fines (LH210), including both blends 1 and 2, are comparable to the peaks of pure $\alpha$-lactose monohydrate. As such, the higher $\gamma_{S V}^{d}$ measured for LH210 cannot be explained by $\beta$-lactose content.

Fig. 5 and Fig. 6 show the $\gamma_{S V}^{d}$ and $\Delta G_{A B}^{0}$ distributions as a function of $n / n_{m}$ respectively. The $\gamma_{S V}^{d}$ for zero surface coverage (Fig. 5) is calculated based on infinite dilution IGC, whereby injection size of alkane probes is held constant at $P / P_{0}=0.03$. We obtain values: $\mathrm{LH} 210=$ $47.53 \mathrm{~mJ} / \mathrm{m}^{2}$, Blend $2=46.86 \mathrm{~mJ} / \mathrm{m}^{2}$, Blend $1=46.54 \mathrm{~mJ} / \mathrm{m}^{2}, \mathrm{LH} 250=45.18 \mathrm{~mJ} / \mathrm{m}^{2}$ and LH100 $=41.93 \mathrm{~mJ} / \mathrm{m}^{2}$.

When the fines (LH210) were blended with coarse lactose (LH250) as in Blend 1 and Blend 2, it is expected that the surface energy of the system would increase with increasing fines 
content because the fines posses both higher $\gamma_{S V}^{d}$ and $\Delta G_{A B}^{0}$. This is exactly the case for Blend 1 and Blend 2 as shown in Fig. 5 and Fig. 6. In the case of Blend 1, the introduction of fines shifted the $\gamma_{S V}^{d}$ profile of LH250 from approximately $42 \mathrm{~mJ} / \mathrm{m}^{2}$ to about $43 \mathrm{~mJ} / \mathrm{m}^{2}$, an increase of $\sim 2.5 \%$, at $n / n_{m}=0.10$, whereas the Blend 2 sample was shifted to $\sim 44 \mathrm{~mJ} / \mathrm{m}^{2}(\sim 5 \%$ increase), resulting in a surface energy value close to that of LH210. This indicates that surfaces of the coarse lactose (LH250) were replaced by surfaces of the fines (LH210), with greater propensity for Blend 2 than Blend 1, due to the higher loading of fine particles. It is interesting to note from the surface energy profiles that the blending of fines did not decrease the overall surface energy of the coarse lactose, but actually increased the overall adhesion property of the blend due to the fact that the fines possess much higher surface energy. As the high energy fines were blended with low energy coarse carrier, the fines seem to have adhered together to form clusters of fines as well as adhering to the specific regions of the coarse lactose (Fig. 4), suggesting that surfaces with high energy or binding characteristics do seem to adhere together. The blending of fines to LH250 resulting in an increase in $\Delta G_{A B}^{0}$ values, resulting in the blends possessing characteristics of LH210.

The current results provide the first evidence that the surface energetics of both fine and coarse lactose is an important parameter in ternary inhalation formulation. The inclusion of higher energy fines to coarse carrier will increase the drug-carrier adhesion interactions, because surfaces are replaced by those having stronger adhesion characteristics. Fines would passivate high energy surfaces only if the fines possess smaller surface energy compared to the coarse carrier. However, the increase of drug-carrier adhesion was reported to increase inhalation performance of drug (Cline and Dalby, 2002; Tong et al., 2006), and it seems that more 
understanding is required in order to understand the relative influence of drug-carrier adhesion and drug-drug cohesion of the drug in the performance of inhalation formulations. 


\section{Conclusions}

The surface properties of lactose crystals were found to be heterogeneous and the surface energy varies from $40 \mathrm{~mJ} / \mathrm{m}^{2}$ to $48 \mathrm{~mJ} / \mathrm{m}^{2}$. The unmilled coarse crystalline lactose sample (LH100) showed less heterogeneity than the milled sample (LH250). The loaded fines (LH210) have a higher energy than the coarse lactose. The amount of fines in Blend 1 was not able to alter the surface energy heterogeneity, whereas sample Blend 2 showed adequate loading of fines to obtain a relatively homogeneous surface. The current surface energy distribution methodology was able to distinguish the differences between these lactose samples. Surfaces of the coarse lactose were shown to be replaced by surfaces of the fines. We conclude that the effects of fines result in an overall increase in surface energy. 


\section{Nomenclature}

\begin{tabular}{|c|c|}
\hline$a_{m}$ & molecular cross-sectional area of adsorbates \\
\hline$A$ & chromatogram area \\
\hline$F$ & carrier gas exit flowrate \\
\hline$\Delta G^{0}$ & standard energy of adsorption \\
\hline$\Delta G_{A B}^{0}$ & standard polar (acid-base) adsorption energy \\
\hline$h$ & chromatogram peak height \\
\hline$j$ & James-Martin correction factor \\
\hline$m$ & sample mass \\
\hline$n$ & amount adsorbed \\
\hline$n_{m}$ & monolayer coverage \\
\hline$N_{A}$ & Avogadro’s number \\
\hline$P$ & pressure \\
\hline$P_{0}$ & saturation pressure \\
\hline$R$ & universal gas constant \\
\hline$S S A_{B E T}$ & specific surface area from nitrogen adsorption isotherm \\
\hline$t_{R}$ & retention time \\
\hline$t_{0}$ & dead time \\
\hline$T$ & temperature \\
\hline$T_{\text {Loop }}$ & injection loop temperature \\
\hline$V_{\text {Loop }}$ & injection loop volume \\
\hline$V_{N}$ & net retention volume \\
\hline$W_{A}$ & work of adhesion \\
\hline$\gamma_{L V}^{d}$ & liquid-vapour dispersive surface energy component \\
\hline$\gamma_{L V}^{p}$ & liquid-vapour polar surface energy component \\
\hline$\gamma_{S V}^{d}$ & solid-vapour dispersive surface energy component \\
\hline$\gamma_{S V}^{p}$ & solid-vapour polar surface energy component \\
\hline$\theta$ & surface coverage \\
\hline
\end{tabular}




\section{References}

Cline, D., Dalby, R., 2002. Predicting the quality of powders for inhalation from surface energy and area. Pharm. Res. 19, 1274-1277.

Del Valle, E.M.M., Galan, M.A., Carbonell, R.G., 2009. Drug delivery technologies: the way forward in the new decade. Ind. Eng. Chem. Res. 48, 2475-2486.

Haque, K., Roos, Y.H., 2005. Crystallization and X-ray diffraction of spray-dried and freezedried amorphous lactose. Carbohydr. Res. 340, 293-301.

Heng, J.Y.Y., Pearse, D.F., Wilson, D.A., Williams, D.R., 2006. Characterisation of Solid State Materials Using Vapour Sorption Methods. In: Zakrzewski, A. and Zakrzewski, M. (Ed.), Solid State Characterisation of Pharmaceuticals, ASSA Inc., Danbury, pp. 449-472.

Ho, R., Hinder, S.J., Watts, J.F., Dilworth, S.E., Williams, D.R., Heng, J.Y.Y., 2009a. Determination of surface heterogeneity of D-mannitol by sessile drop contact angle and finite concentration inverse gas chromatography. Int. J. Pharm. (Accepted)

Ho, R., Wilson, D.A., Heng, J.Y.Y., 2009b. Crystal habits and the variation in surface energy heterogeneity. Cryst. Growth Des. 9, 4907-4911.

Jones, M.D., Price, R., 2006. The influence of fine excipient particles on the performance of carrier-based dry powder inhalation formulations. Pharm. Res. 23, 1665-1674.

Louey, M.D., Stewart, P.J., 2002. Particle interactions involved in aerosol dispersion of ternary interactive mixtures. Pharm. Res. 19, 1524-1531.

Owens, D.K., Wendt, R.C., 1969. Estimation of the surface free energy of polymers. J. Appl. Polym. Sci. 13, 1741-1747.

Podczeck, F., 1998. Adhesion forces in interactive powder mixtures of a micronised drug and carrier particles of various particle size distributions. J. Adh. Sci. Tech. 12, 1323-1339.

Raghavan, S.L., Ristic, R.I., Sheen, D.B., Sherwood, J.N., 2001. The bulk crystallisation of alpha-lactose monohydrate from aqueous solution. J. Pharm. Sci. 90, 823-832.

Schultz, J., Lavielle, L., Martin, C., 1987. The role of the interface in carbon fibre-epoxy composites J. Adhes. 23, 45-60.

Shur, J., Harris, H., Jones, M.D., Kaerger, J.S., Price, R., 2008. The role of fines in the modification of the fluidisation and dispersion mechanism within dry powder inhaler formulations. Pharm. Res. 25, 1931-1940. 
Thielmann, F., Burnett, D.J., Heng, J.Y.Y., 2007. Determination of the surface energy distributions of different processed lactose. Drug Dev. Ind. Pharm. 33, 1240-1253.

Timsina, M.P., Martin, G.P., Marriott, C., Ganderton, D., Yianneskis, M., 1994. Drug-delivery to the respiratory-tract using dry powder inhalers. Int. J. Pharm. 101, 1-13.

Tong, H.H.Y., Shekunov, B.Y., York, P., Chow, A.H.L., 2006. Predicting the aerosol performance of dry powder inhalation formulations by interparticulate interaction analysis using inverse gas chromatography. J. Pharm. Sci. 95, 228-233.

Yla-Maihaniemi, P.P., Heng, J.Y.Y., Thielmann, F., Williams, D.R., 2008. Inverse gas chromatographic method for measuring the dispersive surface energy distribution for particulates. Langmuir 24, 9551-9557.

Zeng, X.M., Martin, G.P., Tee, S.K., Abu Ghoush, A., Marriott, C., 1999. Effects of particle size and adding sequence of fine lactose on the deposition of salbutamol sulphate from a dry powder formulation. Int. J. Pharm. 182, 133-144.

Zeng, X.M., Pandhal, K.H., Martin, G.P., 2000. The influence of lactose carrier on the content homogeneity and dispersibility of beclomethasone dipropionate from dry powder aerosols. Int. J. Pharm. 197, 41-52. 


\section{Figure Legends}

Fig. 1. Passivation of high energy sites: Fines are first blended with coarse carrier particles before blending with the particles of the active drug.

Fig. 2. Determination of $\gamma_{S V}^{d}$ and $\Delta G_{A B}^{0}$ from a plot of net retention volume versus vapour surface property

Fig. 3. Surface energy heterogeneity determination.

Fig. 4. SEM images of lactose crystals and blends of fine and coarse lactose.

Fig. 5. Dispersive surface energy distributions (versus surface coverage) for all lactose samples used in the study.

Fig. 6. $\Delta G_{A B}^{0}$ (ethanol) distributions (versus surface coverage) for all lactose samples used in the study. 


\section{Table 1}

Details of the lactose samples used in the current investigation.

\begin{tabular}{|l|l|l|}
\hline Sample & Code & Description \\
\hline Lactohale ${ }^{\circledR}$ LH100 & LH100 & Unmilled, crystalline \\
\hline Lactohale ${ }^{\circledR}$ LH210 & LH210 & Milled \\
\hline Lactohale ${ }^{\circledR}$ LH250 & LH250 & Crystalline, gently milled with fines removed \\
\hline $\begin{array}{l}\text { Lactohale }{ }^{\circledR} \text { LH250 with } \\
\text { Lactohale }{ }^{\circledR} \text { LH210 }\end{array}$ & Blend 1 & LH250 and LH210 in 82:18 ratio \\
\hline $\begin{array}{l}\text { Lactohale }{ }^{\circledR} \text { LH250 with } \\
\text { Lactohale }{ }^{\circledR} \text { LH210 }\end{array}$ & Blend 2 & LH250 and LH210 in 70:30 ratio \\
\hline
\end{tabular}

\section{Table 2}

Particle size and BET specific surface area of the lactose samples.

\begin{tabular}{|c|c|c|c|c|l|}
\hline Sample & \multicolumn{3}{|l|}{ Particle Size (based on an equivalent sphere) } & $\begin{array}{l}\text { BET Specific } \\
\text { Surface Area } \\
\\
\end{array}$ \\
& $\mathrm{d}_{10}(\mu \mathrm{m})$ & $\mathrm{d}_{50}(\mu \mathrm{m})$ & $\mathrm{d}_{90}(\mu \mathrm{m})$ & $\%<15 \mu \mathrm{m}$ & \\
\hline LH100 & 59.0 & 149.1 & 228.5 & 2.65 & $0.1164 \pm 0.0016$ \\
\hline LH210 & 3.2 & 18.0 & 48.0 & 42.2 & $0.8120 \pm 0.0036$ \\
\hline LH250 & 36.6 & 80.7 & 138.2 & 3.2 & $0.1893 \pm 0.0016$ \\
\hline Blend 1 & 10.6 & 65.2 & 131.3 & 13.0 & $0.2785 \pm 0.0020$ \\
\hline Blend 2 & 7.2 & 53.9 & 124.2 & 18.2 & $0.3510 \pm 0.0023$ \\
\hline
\end{tabular}


Fig. 1. Passivation of high energy sites: Fines are first blended with coarse carrier particles before blending with the particles of the active drug.

Fines And Coarse Carrier Particles Blending

Fine excipient particle

High energy
sites
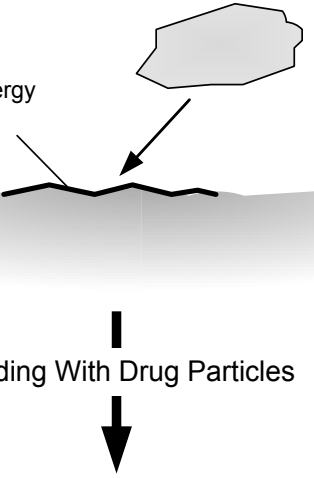

Blending With Drug Particles

$\downarrow$

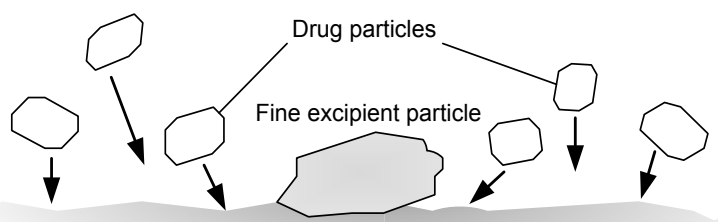


Fig. 2. Determination of $\gamma_{S V}^{d}$ and $\Delta G_{A B}^{0}$ from a plot of net retention volume versus vapour surface property via the Schultz approach.

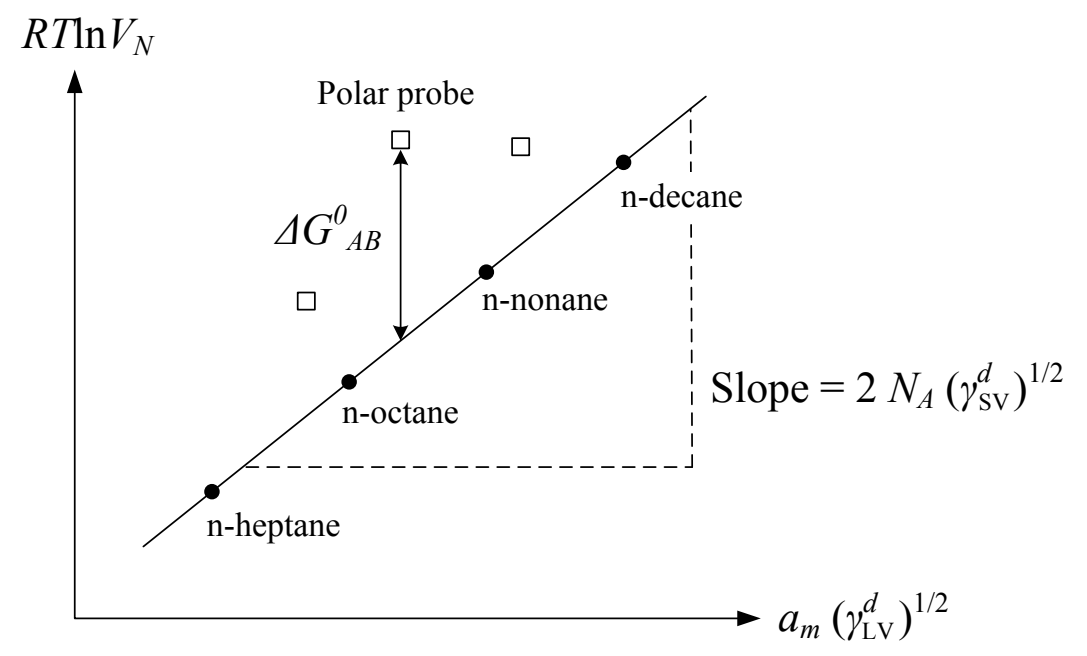


Fig. 3. Surface energy heterogeneity determination.

1. Monolayer Coverage Determination

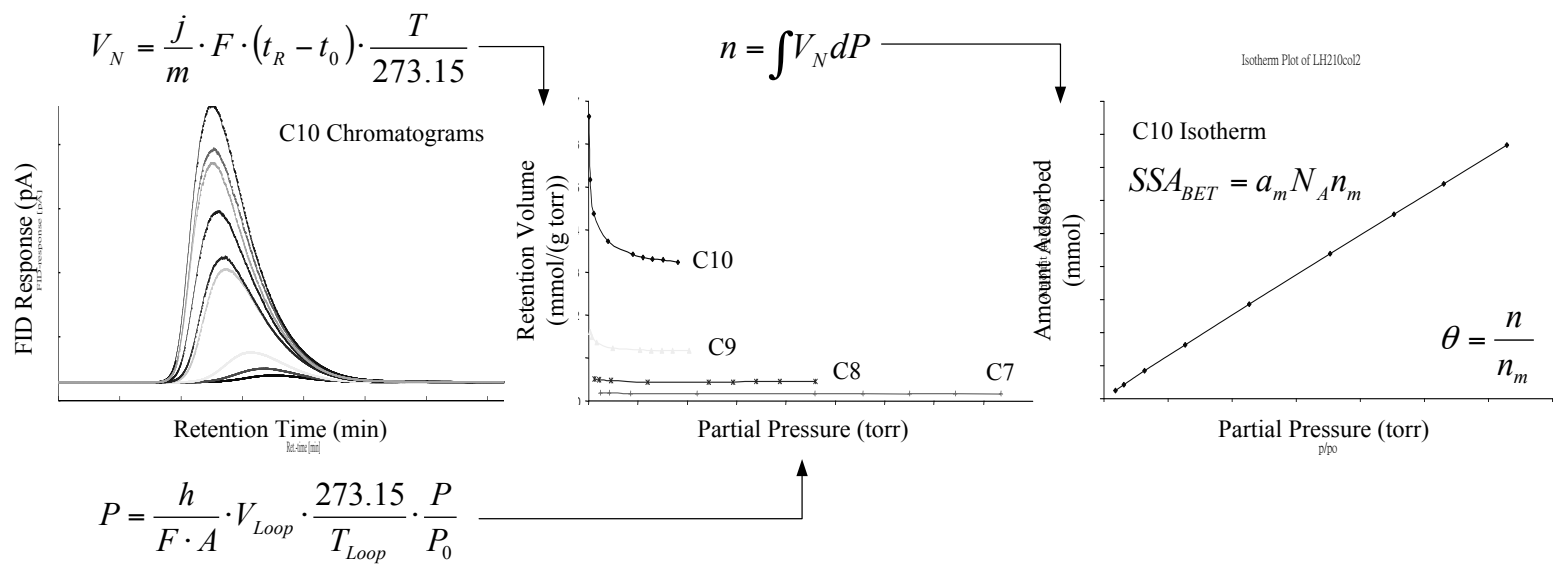

2. Dispersive Surface Energy Profile
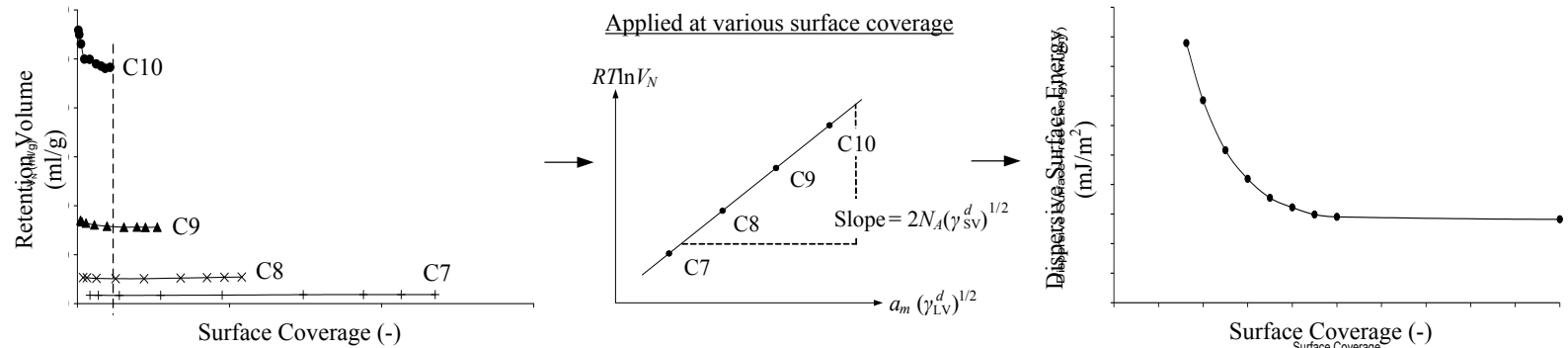

3. Polar (Acid-base) Energy of Adsorption Profile
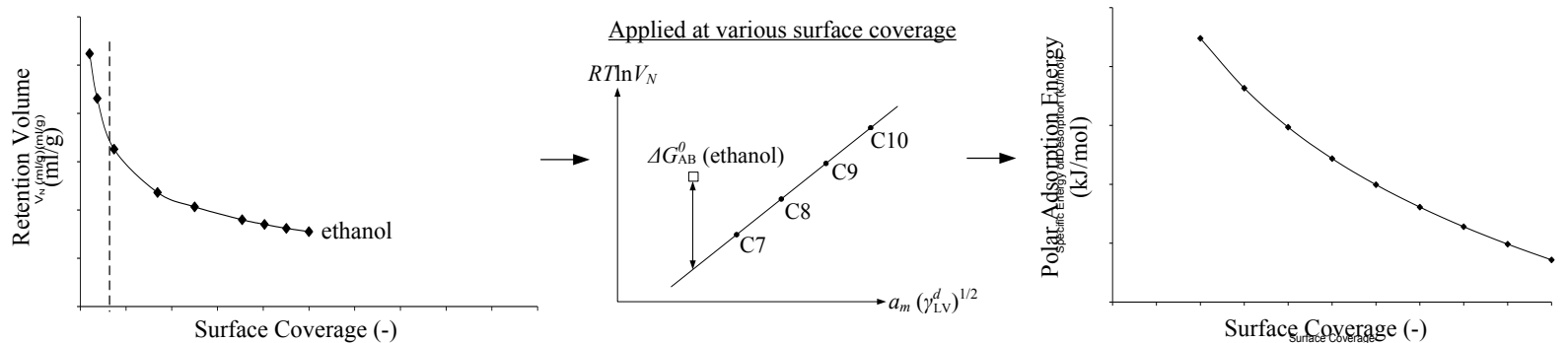
Fig. 4. SEM images of lactose crystals and blends of fine and coarse lactose.
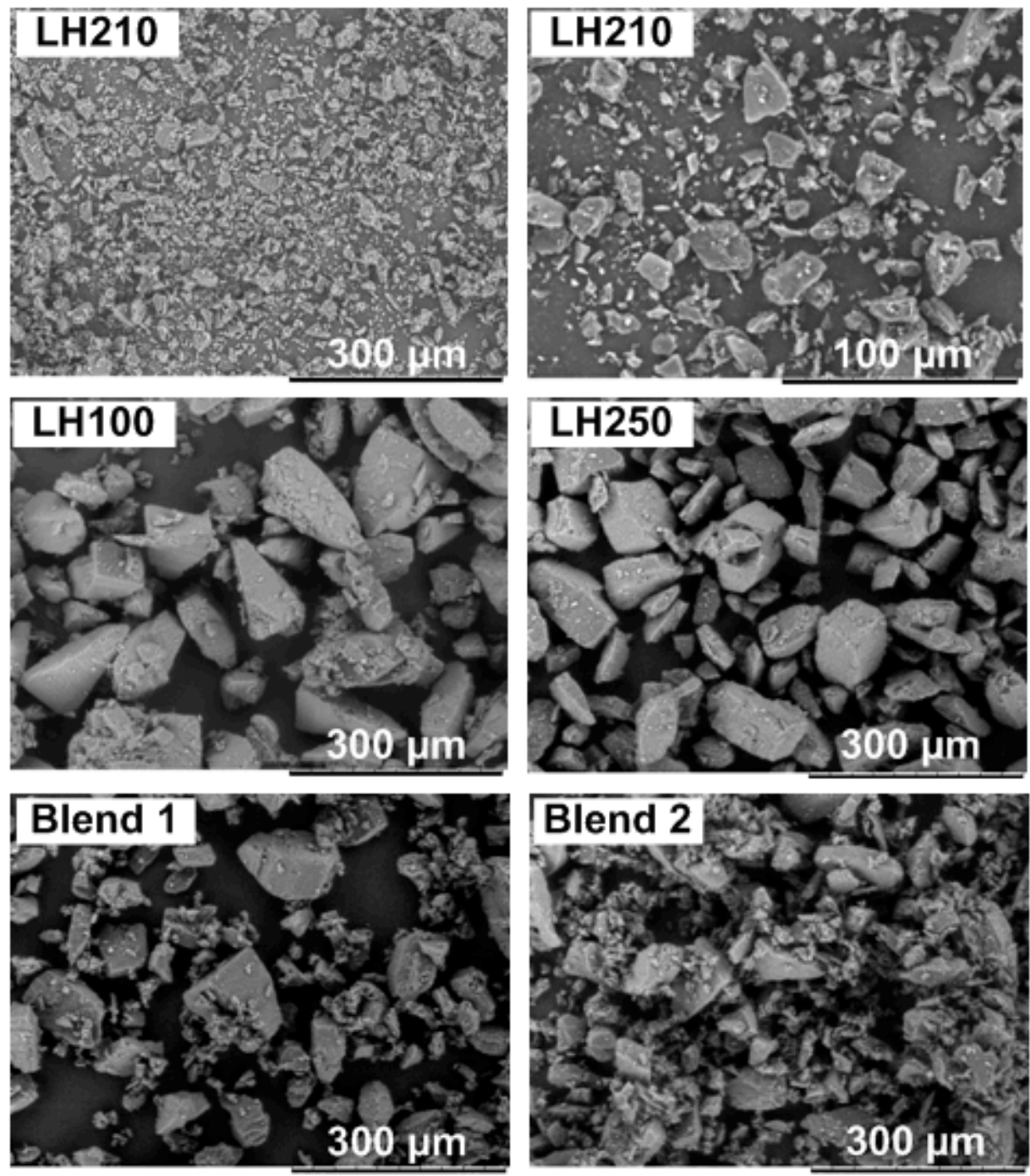
Fig. 5. Dispersive surface energy distributions (versus surface coverage) for all lactose samples used in the study.

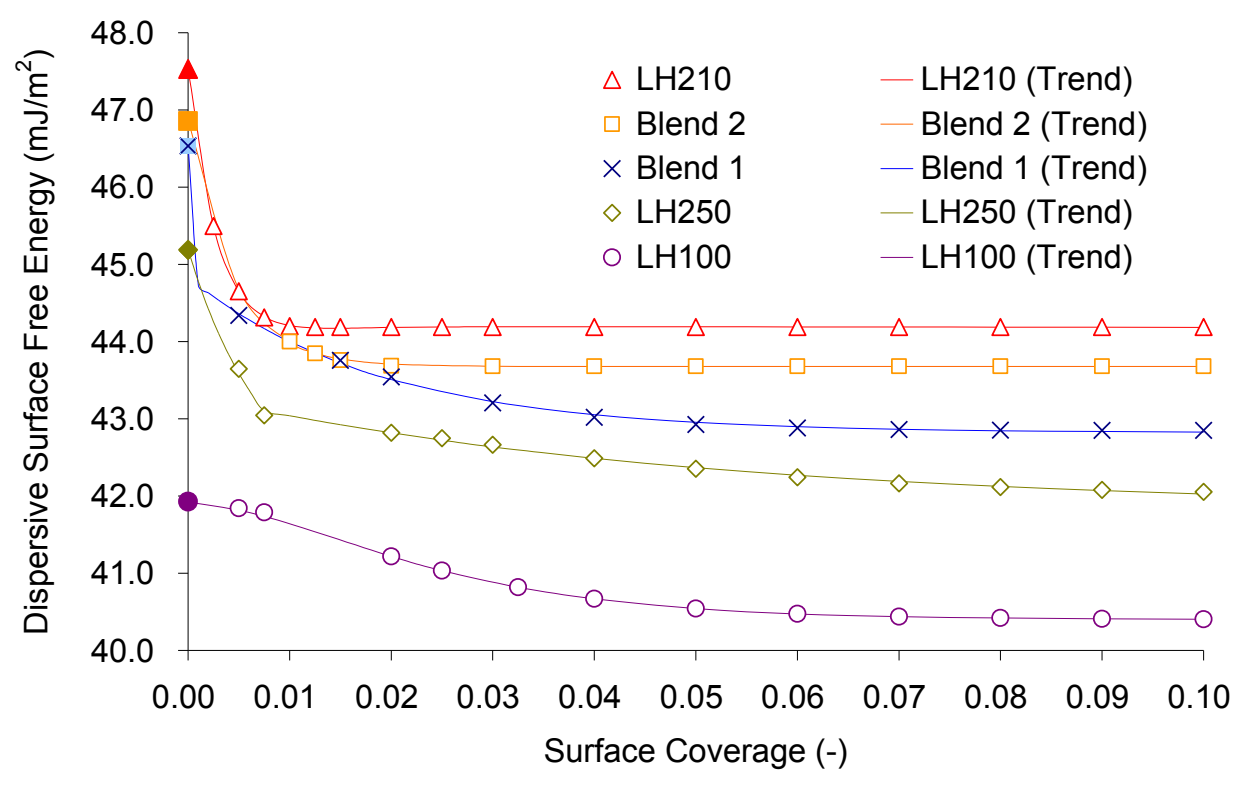


Fig. 6. $\Delta G_{A B}^{0}$ (ethanol) distributions (versus surface coverage) for all lactose samples used in the study.

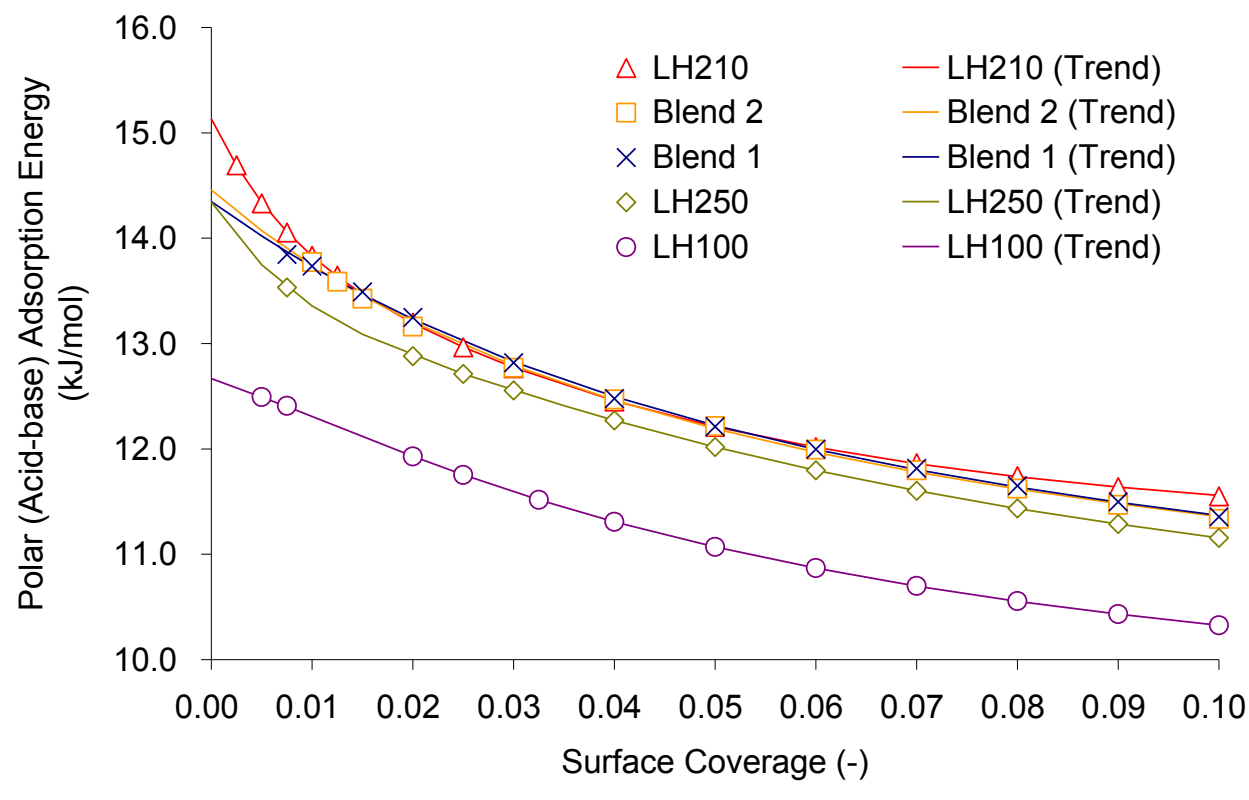

